\title{
Method of Determining a Nominal Index Value for the Polytropic Expansion Process of Supercritical Carbon Dioxide in Piston-Cylinder Devices
}

\author{
C.M. McNamara and K.R. Anderson* \\ California State Polytechnic University at Pomona \\ Mechanical Engineering Department \\ 3801 West Temple Ave, Pomona, CA, 91768, USA \\ Solar Thermal Alternative Renewable Energy Lab \\ E-mail: ${ }^{1}$ kranderson@ @csupomona.edu
}

\begin{abstract}
The polytropic process is a widely used pressure model for predicting the nature of pressure and volume states in expansion and compression systems. The two variable nature of this model typically leads to an open ended approach with heavy reliance on mechanism evaluation and potential extended design iterations in the prototyping phase. Through a fundamental examination of the polytropic derivation from the energy transfer ratio assumption, in conjunction with repeated application of elementary thermodynamic principles, including First Law analysis, moving boundary work, and substance property evaluation, a solution to a refined nominal polytropic index can be found. The derivation can provide valuable insight to the polytropic process itself and address the issue of bounding a complex system with a reasonable choice of theoretical system input. With the aid of the method outlined in this paper, researchers will be enabled with a tool to better predict the polytropic expansion coefficient which finds widespread use in thermodynamic modeling and analysis. To this end, the method is used herein to predict a polytropic index of $n=1.306$ and $k=1.615$ for the specific heat ratio for a SCO2 expansion engine operating between $20 \mathrm{MPa}$ and $9.2 \mathrm{MPa}$.
\end{abstract}

\section{Keywords: Polytropic expansion; supercritical carbon dioxide; thermodynamics modeling; cycle analysis}

\section{Introduction}

The motivation of this current paper stems from a desire to more accurately predict the value of the polytropic exponent used in the modeling of a Supercritical Carbon Dioxide (SCO2) Waste Heat Recovery Rankine Engine Cycle. The current paper is motivated by the study of Anderson et al. [1] whereby the polytropic model is used to predict the expansion process in a SCO2 Rankine Engine for use in regenerative waste heat to power recovery, and also the work of Anderson et al. [2] wherein the thermodynamic polytropic expansion process is utilized in the modeling and analysis of a lightweight, high specific power, two-stroke, polygon engine.

Figure 1 shows the SCO2 Heat Recovery cycle being studied herein as presented by Anderson et al. [1]. As outlined in [1], in order to determine the most efficient operating point, a parametric analysis is performed using first order thermodynamics. For the prototype cycle, limits are chosen to be $35^{\circ} \mathrm{C}$ and pressure above $7.4 \mathrm{MPa}$ to maintain the fluid above the critical point and avoid twophase flow. Temperature on the high side is dictated by input from a low quality heat source, which generally originates at $200{ }^{\circ} \mathrm{C}-500{ }^{\circ} \mathrm{C}$. These were the design factors which constrained our work to be between the low and high side pressures of $12.5 \mathrm{MPa}$ and $20 \mathrm{MPa}$, respectively. The expansion process between state 4 and 5 of the pressureenthalpy state diagram of Figure 1 is modeled using the polytropic assumption. The modeling of this process is the main focus of this current paper. The waste heat regenerative Rankine cycle of Figure 1 is composed of six main hardware components. As indicated in Figure 1, the
$\mathrm{SCO} 2$ cycle starts at state point 1 , corresponding to a low side pressure above $7.5 \mathrm{MPa}$ and a low side temperature of $35{ }^{\circ} \mathrm{C}$, slightly above the critical point of $\mathrm{CO}_{2}$. After compression the cycle reaches state point 2, where the SCO2 is brought to the high-side pressure of $20 \mathrm{MPa}$ and a temperature of $36{ }^{\circ} \mathrm{C}$, approximately $1{ }^{\circ} \mathrm{C}$ higher than the pre-compressed state. An internal heat exchanger process between state points 2-3 then heats the pressurized $\mathrm{SCO} 2$ by exchange with low-pressure, post-expansion SCO2. After exiting the internal heat exchanger at state points 3 , the SCO2 is heated by a second heat exchanger where addition is done via waste heat, raising the temperature to its ultimate value of approximately $200{ }^{\circ} \mathrm{C}$ and leaving the $\mathrm{SCO} 2$ at state point 4 . The heated and pressurized $\mathrm{SCO} 2$ is then expanded between state points 4 and 5 in an expansion device which is tied to generator to produce rotational energy and eventually electricity. The rotational energy is converted to electricity by coupling the expander's output shaft to a permanent magnet alternator. The fluid exits at a low-side pressure of $12.4 \mathrm{MPa}$ and a temperature of $163{ }^{\circ} \mathrm{C}$ to state point 5 . The expanded fluid is then run through the internal heat exchanger where it is cooled by high-pressure, pre-expansion $\mathrm{SCO} 2$ and then arrives at state point 6 . The cooled supercritical $\mathrm{CO} 2$ is finally passed through a radiator where it exits at the pre-compressed pressure and temperature and then returns to state point 1 to repeat the cycle.

Figures 2-4 show the polytropic expansion process under the context of the current study in pressure/volume, temperature/entropy, and pressure/enthalpy state diagrams, respectively As shown in Figure 2 the present application 
deals with $\mathrm{SCO} 2$ in the region of high-side pressures on the order of $20 \mathrm{MPa}$, and low-side pressures on the order of 9 $\mathrm{MPa}$, while the specific volume ranges from $0.004 \mathrm{~kg} / \mathrm{m}^{3}$ to $0.007 \mathrm{~kg} / \mathrm{m}^{3}$.

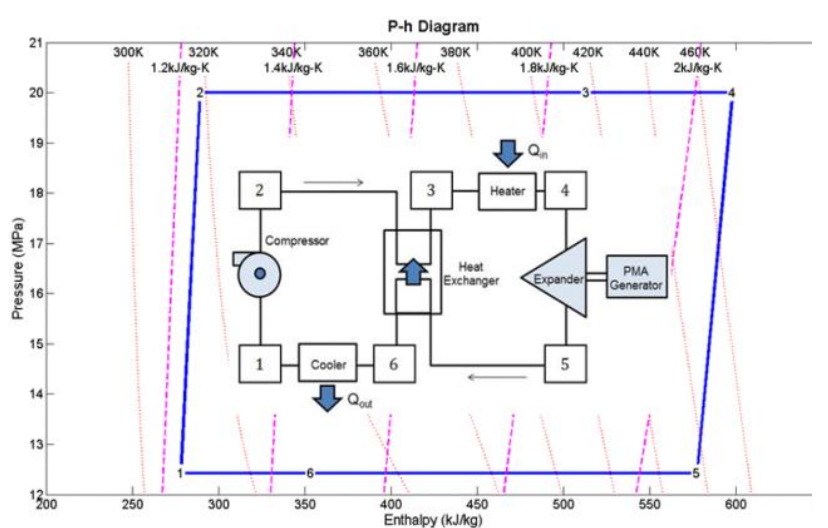

Figure 1. Supercritical carbon dioxide (SCO2) cycle on Mollier diagram.

Figure 3 shows that the temperature range associated with the expansion of the $\mathrm{SCO} 2$ ranges from a high of 470 $\mathrm{K}$ to a low of $390 \mathrm{~K}$ while the entropy increases form 2.05 $\mathrm{kJ} / \mathrm{kg}-\mathrm{K}$ to $2.07 \mathrm{~kg} / \mathrm{kJ}-\mathrm{K}$. Figure 4 shows that the enthalpy at the high-side pressure is on the order of $599 \mathrm{~kJ} / \mathrm{kg}$ and for the low-side pressure, the enthalpy is roughly $555 \mathrm{~kJ} / \mathrm{kg}$.

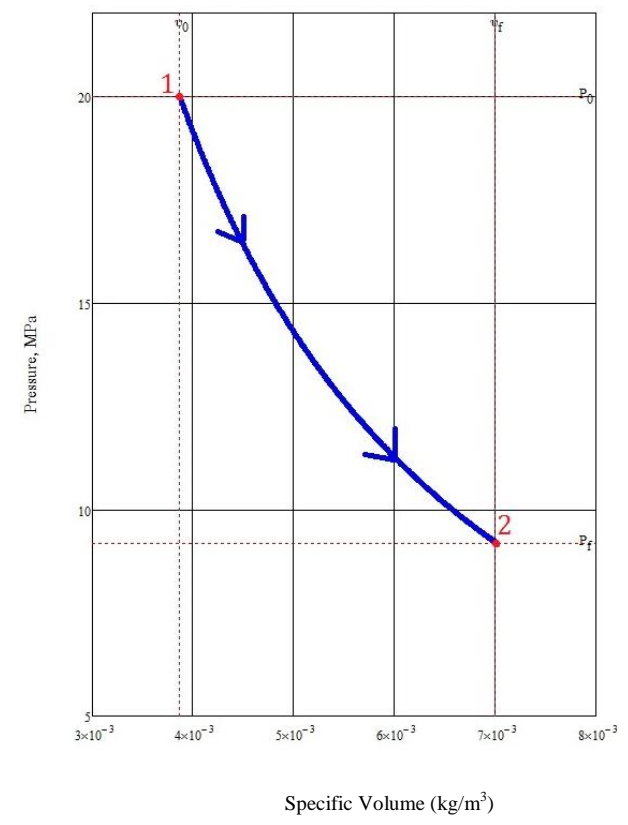

Figure 2. p-v diagram of SCO2 expansion process.

It can be seen from Figures 2-4, the expansion process is non-isentropic, i.e. the entropy does not remain constant, and heat is transferred since the enthalpy value changes. The focus of this current paper is to develop a method to determine the optimum polytropic exponent for use in the expansion process indicated in the cycle of Figure 1. The use of the polytropic coefficient in thermodynamics research is widespread in current day research. Many current investigations rely on the venerable polytropic model as the cornerstone of research and development endeavors.

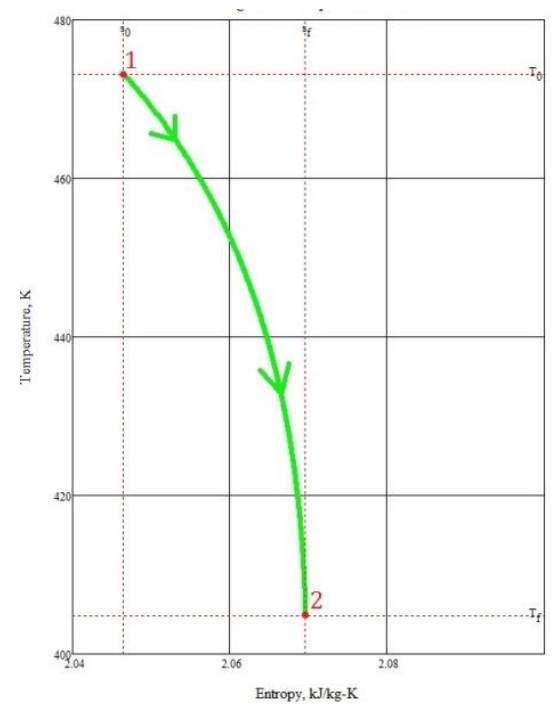

Figure 3. T-s diagram of SCO2 expansion process.

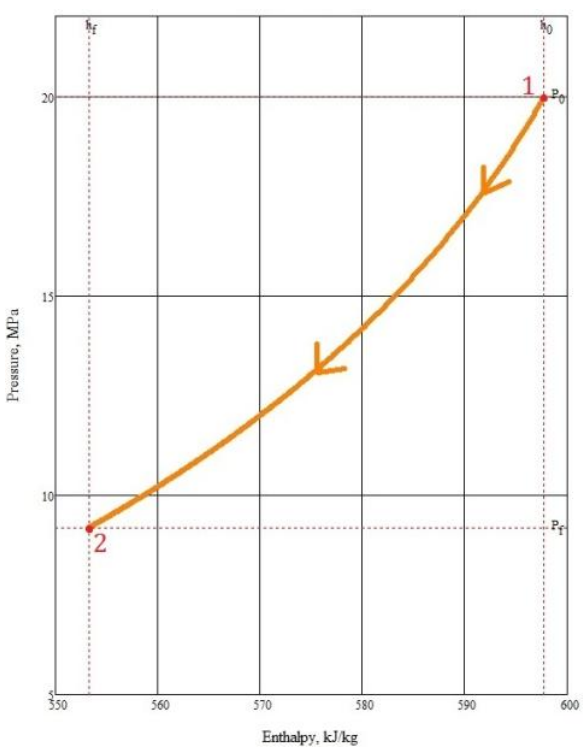

Figure 4. p-h diagram of SCO2 expansion process.

The work of Heiser et al. [3] presents the results of a fundamental, exhaustive analytical and computational examination of the performance of the Brayton propulsion and power cycle employing real air as the working fluid. The analysis of [3] employs a correlation to represent the specific heat at constant pressure of air as a function of temperature and the polytropic efficiency in order to evaluate the overall efficiency of the adiabatic compression and expansion processes. The analytical results of [3] present a useful guidance for thermodynamic engineers. In the study of Lapuerta et al. [4], the polytropic coefficient during the compression cycle of a reciprocating internal combustion engine depends on the instantaneous values for pressure and volume, as well as on their variations. In the work of [4], knowledge of the polytropic coefficient is used to determine whether or not heat flux transferred to the walls, which is a parameter that continues to evade modeling and/or empirical investigations. The research of Zhao et al. [5] proposes a measurement technique based on the dynamic regularity of instantaneous polytropic exponents during discharge of flow in pneumatic components. In the work of [5], a piecewise polynomial fitting curves of instantaneous polytropic exponents is the cornerstone to a new algorithm for the identification of flow 
rate characteristics is proposed. The identification errors of sonic conductance of the new method developed in [5] are stated to by less than $3 \%$, and the identification errors of critical pressure ratio of the new algorithm given by [5] are less than $15 \%$. In the research of Chang et al. [6], a thermodynamic method of top dead center (TDC) correction in the indicator diagram of diesel engines is presented. The method outlined in [6] is based on the corresponding relationship between the curve for the heat released and the computed polytropic exponent. The experiments of [6] demonstrate that this method can make the real-time correction of TDC for the Diesel engines. From this literature review, we see that the use of the polytropic exponent in fundamental thermodynamic research is still warranted and justified, and its determination is usually typified using a combination of complex numerical modeling in conjunction with the conduction of experiments.

The polytropic pressure model is typically used to model the pressure history in combustion processes due to the fact that the process tends to behave close to that of an adiabatic isentropic process for most conventional fuels [7][10]. In general the polytropic model tends to be attractive as starting point for the analysis of piston cylinder devices due to its ability to include, rather than neglect the effects of heat transfer. This is desirable as it allows the designer to first approach a problem involving complex heat transfer phenomenon and work production without explicitly modeling and quantifying the heat transfer. Moreover, due to the nature of the pressure model, it lends itself to easy iteration and manipulation as well as exclusion of physically improbable results giving the designer a range of expected behaviors. Polytropic index values and the types of processes they correspond to are well known per the work of Christians [11]. This current paper seeks to narrow the range of expected behaviors to that of a single behavior model that shortens the initial modeling process and gives consistent results. Thus eliminating valuable time spent in the laboratory. This is done by evaluating the polytropic process on a fundamental thermodynamic level and applying its assumptions throughout the modeling process. While it is recognized that empirical data will dictate the actual polytropic expansion coefficient, with the aid of the method outlined in this paper, researchers will be enabled with a tool to model and predict the polytropic expansion coefficient prior to engaging in sometimes cost prohibitive testing.

The development of the polytropic model from its fundamental thermodynamics basis is outlined in the work of Christians [11]. What commonly is presented as simply a pressure model is derived and must be consistent with a more basic assumption, i.e. that of a constant energy transfer ratio. The work of [11] gives a polytropic process as

$P v^{n}=C$

$n=(1-k) \tau+k$

$\tau \equiv \frac{\delta q}{\delta w}=\frac{n-k}{1-k}$

Here Eq. (1) represents the familiar polytropic process pressure model, where Eqs. (2) and (3) define the energy transfer ratio, $\tau$ and the subsequent relation of the polytropic index, $n$ and isentropic index, $k$ (or heat capacity ratio) to it. These relations will prove important to the development of a method for determining the proper value of the polytropic index, $n$. It should be noted that for the polytropic pressure model, this energy transfer ratio must be constant throughout the process [11].

\section{Methodology \\ 2.1 Derivation}

Working with the derivations proposed by [7], it is applied using a First Law analysis to a piston cylinder device. If a polytropic process model is to be used, the pressure model can be shown to be Eq. (4)

$P(V)=P_{0}\left(\frac{V_{0}}{V}\right)^{n}$

which is done by using the initial cylinder conditions. Using the terminology of internal combustion engines, the two initial values are typically known where the initial volume, $\mathrm{V}_{0}$ is the top dead center volume and $\mathrm{P}_{0}$ is the intake pressure. Additionally the volume of a piston cylinder device as a function of stroke can be shown as given in Eq. (5)

$V(x)=V_{0}+A x$

where $A$ is the piston cross-sectional area and $x$ is the distance traveled along the stroke. Substituting this formula in to Eq. (4) affords

$P=P_{0}\left(\frac{V_{0}}{V_{0}+A x}\right)^{n}$

whereby

$P=P_{0}(1+\zeta x)^{-n}$

and

$\zeta=\frac{A}{V_{0}}$

Here $\zeta$ is the bore to top dead center volume ratio Eq. (7) gives us a convenient explicit formula for pressure in the cylinder as a function of stroke. This can be evaluated as moving boundary work to find an explicit formula for work done by the fluid over the process

$W=\int F d x=\int P A d x=\int_{0}^{s} P_{0}(1+\zeta x)^{-n} d x$

$=\frac{P_{0} V_{0}}{1-n}\left[(\zeta s+1)^{1-n}-1\right]$

where $s$ is the stroke length and the trivial case of $n=-1$ has been omitted from discussion. The explicit formula for work allows application to the First Law of both Eqs. (3) and (9). Two cases must be considered for the case of the First Law for piston-cylinder devices, for net heat transfer to or away from the system. For the case of heat transferred to the system this is given by Eqs. (10)-(18) as shown below

$Q-W=\Delta U$

$W(\tau-1)=\Delta U$ 
$W\left(\frac{n-1}{1-k}-1\right)=\Delta U$

$\frac{n-1}{1-n} P_{0} V_{0}\left[(\zeta s+1)^{1-n}-1\right]=m\left(u_{f}-u_{0}\right)$

$\frac{n-1}{1-n} P_{0} V_{0}\left[(\zeta s+1)^{1-n}-1\right]=\rho_{0} V_{0}\left(u_{f}-u_{0}\right)$

$n=1-\frac{\ln \left[1-\left(\frac{\rho_{0}}{P_{0}}\right)\left(\frac{u_{f}-u_{0}}{1-k}\right)\right]}{\ln (1+\zeta s)}$

$\alpha\left(u_{f}\right)=1-\left(\frac{\rho_{0}}{P_{0}}\right)\left(\frac{u_{f}-u_{0}}{1-k}\right)$

$\beta=1+\zeta s$

$n\left(u_{f}\right)=1-\frac{\ln \left(\alpha\left(u_{f}\right)\right)}{\ln \beta}$

Eqs. (15) and (18) provide a closed form solution for the polytropic index for a piston cylinder device with a net heat transfer to the working fluid. This solution would represent the polytropic index for a typical combustion process. For the case of net heat transfer from the fluid, returning to the First Law and changing the direction of the heat transfer given by Eqs. (19)-(27) below

$-Q-W=\Delta U$

$W(-\tau-1)=\Delta U$

$W\left(-\frac{n-1}{1-k}-1\right)=\Delta U$

$\frac{P_{0} V_{0}}{1-n}\left[(\zeta s+1)^{1-n}-1\right]\left(\frac{n-2 k+1}{1-k}\right)=m\left(u_{f}-u_{0}\right)$

$\frac{P_{0} V_{0}}{1-n}\left[(\zeta s+1)^{1-n}-1\right]\left(\frac{n-2 k+1}{1-k}\right)=\rho_{0} V_{0}\left(u_{f}-u_{0}\right)$

$\left(\frac{n-2 k+1}{1-n}\right)\left[(1+\zeta s)^{1-n}-1\right]=\left(\frac{\rho_{0}}{P_{0}}\right)\left(u_{f}-u_{0}\right)(1-k)$

$\varphi(n)=\left(\frac{n-2 k+1}{1-n}\right)\left[(1+\zeta s)^{1-n}-1\right]$

$\Omega\left(u_{f}\right)=\left(\frac{\rho_{0}}{P_{0}}\right)\left(u_{f}-u_{0}\right)(1-k)$

$\varphi(n)=\Omega\left(u_{f}\right)$

Equations (24) through (27) represent the relation between the polytropic index and the final internal energy state for a piston cylinder with a net heat loss in the same manner that Eq. (18) does for a device with a net heat gain. Although Eq. (18) is a closed form solution and its counterpart Eq. (27) is not, further discussion will show that this mathematical convenience does not serve such a valuable role as one would assume for determining the value of $n$.

An examination of the relations obtained reveals that an apparent guess must still be made to determine the polytropic index due to the reliance on the final state of internal energy. This however can be circumvented in the application of the polytropic model to piston cylinder devices. Noting that all initial state properties are known, bounding the problem from the process beginning, the final state may be obtained via state property relations. For any piston cylinder device, the volume model presented in Eq. (5) applies with disregard of the piston head shape as this will contribute to the top dead center volume. Likewise the amount of mass present in the chamber is known from the initial variables

$m=\rho_{0} V_{0}$

which is a constant throughout the process. Therefore, the density at any point of the process is known by applying the definition of density and using Eq. (5) and Eq. (28) gives

$\rho(x)=\frac{m}{V(x)}=\frac{m}{V_{0}+A x}$

Therefore the final density at the end of the stroke is then defined as

$\rho_{f}=\rho(s)$

and the final volume is simply

$V_{f}=V(s)$

From basic thermodynamic theory, the state of a substance is known when two independent, intensive state properties are known [12]. Therefore mathematically to determine any substance property it is required that a property, $\theta$ be defined as

$\theta=\left\{\begin{array}{l}F(X, Y) ; \text { State outside saturation curve } \\ F(X, Y, Z) ; \text { State inside saturation curve }\end{array}\right.$

where $X, Y$, and $\mathrm{Z}$ are any valid combination of state properties. For the operation of a typical piston cylinder device, two-phase flow is undesirable for operation and virtually any working fluid will be operating far into the gaseous region of a substance's thermodynamic profile. Therefore the analysis is restricted to the primary definition of Eq. (33). It is noted that for determining the polytropic index, the required function is that for internal energy, $u$, which may be defined in terms of any other two independent state variables. Noting that the pressure model being used must reflect the same behavior of the working fluid from its known properties, the following constraints of "Eqs. (33)-(34)" must apply

$P\left(\rho_{f}, X_{f}\right)=P_{0}\left(\frac{V_{0}}{V_{f}}\right)^{n}$

$u\left(\rho_{f}, X_{f}\right)=u_{f}$

which is obtained by equating the pressure model to a surface fit of known pressure data for the working fluid. An analogous process is done for the internal energy. Since the only variable to be solved for is the final value of the property $X$ (which may be any property excluding pressure and internal energy), this final value may be solved for and then substituted back in the developed relations to determine the final internal energy state and therefore the polytropic index.

The multivariable functions for pressure and internal energy may are readily prepared using the computational software MathCAD [13] to create two dimensional splines from available property data made available by sources 
such as the National Institute for Standards and Technology (NIST) [14]. These functions may then be used to easily solve the complex relations quickly. In the following discussion, computer computation should be a prevalent method for determining the most accurate solution when dealing with fluids operating over a wide range of operating conditions.

\subsection{Model Limitations and Accuracy Considerations}

The primary dimensions for the paper are summarized in Table 1. A fundamental shortcoming of the polytropic model lies in the assumptions that are used in its development. The following assumptions are made during the development

1) Energy transfer ratio is constant throughout process.

2) Calorically perfect gas (constant specific heats and therefore isentropic index).

3) Compressibility factor constant throughout process.

4) System is internally reversible.

The first assumption is necessary to begin the discussion of the polytropic process in general. The second assumption is a common one to make in many thermodynamic simplified analyses but is known to generally be untrue unless the process occurs over small ranges of operating parameters. This likewise is the case for the third assumption. Finally the fourth assumption is never a real world possibility. The scope however is to develop an approximate model. The sources of largest error will be those associated with the second and third assumptions.

Turning the attention first to the compressibility factor, it is noted that

1) At very low pressures, gases behave ideally regardless of temperature.

2) At high temperatures, gases behave ideally regardless of pressure.

3) Deviation from ideal gas behavior is greatest near the critical point.

For typical operating ranges of working fluids such as air and other generally ideally-behaving gases, this is a highly valid assumption. For other fluids the specific region of operation should be examined to determine the expected variability of the compressibility factor.

Examining the calorically perfect gas assumption, the largest problem is when the operating range is extended over a large array of conditions. This causes large variations in the isentropic index which is expected to remain constant. In these types of operating conditions, this will be the dominant source of error. A method to reduce this error is to introduce an average of the isentropic index over the expected operating ranges. This can be done by applying the well-known definition of two-dimensional average from calculus given in Eq. (35) as

$$
k_{\text {avg }}=\frac{\int_{P_{a}}^{P_{b} T_{a}} k_{P T}(T, P) d T d P}{\int_{P_{a}} \int_{T_{a}} d T d P}
$$

wherein

$k_{P T}(T, P)=\frac{C_{p, R T}}{C_{v, R T}}$

Since the end state of the process depends on this average, the designer should compare the end result temperatures and pressures to the limits of integration chosen at the beginning of the process and then re-run the process as many times as necessary to reach the designer's level of accepted solution convergence.

\section{Results}

The following parameters were used to illustrate the method of determining a nominal polytropic process index value for the expansion process of outlined in this paper: stroke length $=0.0508 \mathrm{~m}$ ( $2 \mathrm{in})$, piston diameter $=0.0508 \mathrm{~m}$ (2.0 in), top-dead-center (TDC) chamber volume = $0.000253 \mathrm{~m}^{3}\left(15.44 \mathrm{in}^{3}\right)$, bottom-dead-center (BDC) chamber volume $=0.000459 \mathrm{~m}^{3}\left(28.006 \mathrm{in}^{3}\right)$, critical properties of SCO2: $T_{\mathrm{c}}=304.1282 \mathrm{~K}, P_{\mathrm{c}}=7.3773 \mathrm{MPa}$, $\rho_{\mathrm{c}}=467.6 \mathrm{~kg} / \mathrm{m}^{3}$, expansion entry state: $T_{\text {entry }}=473 \mathrm{~K}$, $P_{\text {entry }}=20 \mathrm{MPa}, \rho_{\text {entry }}=285.82 \mathrm{~kg} / \mathrm{m}^{3}$, expansion exit state: $T_{\text {exit }}=436 \mathrm{~K}, P_{\text {exit }}=12.4 \mathrm{MPa}, \rho_{\text {exit }}=176.78 \mathrm{~kg} / \mathrm{m}^{3}$.

Analyzing Carbon Dioxide (CO2) without a specific Equation of State (EOS) for approximation will be difficult for looking up values, especially for automation. To automate, read in tables for desired property interpolation functions. Note that two independent, intensive properties are required to fix state. Since the only time three variables are needed is in the saturated region because $P$ and $T$ are fixed, and the design will not function outside the supercritical region, only data above the critical point will be input. All data files here were created using NIST REFPROP 9.1.0.0 [14] and building an EXCEL spreadsheet SCO2 database. These EXCEL NIST REFPROP SCO2 databases are then read into MathCAD [13], which is used to perform the necessary iterations in finding the nominal polytropic process index value.

In the supercritical state, the isentropic index can vary largely. The supercritical state influences the isentropic index not only via temperature but also via pressure. The polytropic model is derived under assumptions of a calorically perfect gas following the work of Christians [11]. This requires the isentropic index to be constant. This can be approximated by averaging the index over the expected states. Since functions from NIST are available for the heat capacities, $k$ can be defined in the usual manner via Eq. (36), from whence the averaging function defined in Eq. (35) may then be evaluated via numerical quadrature. For $P_{a}=9.2 \mathrm{MPa}, P_{b}=20 \mathrm{MPa}, T_{a}=405 \mathrm{~K}, T_{b}=472 \mathrm{~K}$ (these limits represent the expected range of temperature and pressure to be witnessed by the $\mathrm{SCO} 2$ expansion engine), this procedure affords $k_{\text {avg }}=1.615$ (as discussed in detail below).. It should be mentioned here the limits of integration used in Eq. (35) are the by-product of manual integration of the entire modeling process of the $\mathrm{SCO} 2$ cycle as a whole. Starting with the overall specifications per Anderson et al. [1] the thermodynamic expansion process model was run, the entire $\mathrm{SCO} 2$ was re-run several times with the newly obtained cycle limits from the prior run used to establish new limits on the integral of Eq. (35). This process is continued until convergence is reached.

This being said, it is clear that proper determination of the parametric value $\mathrm{n}$ is complex and must be solved via 
iteration methods provided the problem bounds are set correctly. Assuming that the process is indeed polytropic, the energy transfer ratio must remain constant by definition. As such, the pressure model end state must match the values chosen in the SCO2 NIST database. To locate a result in the SCO2 NIST database other state properties are needed. However, note the final density in the expander is fixed by the stroke length and the required mass. Therefore $V_{\text {final }}=0.000459 \mathrm{~m}^{3}\left(28.006 \mathrm{in}^{3}\right), \mathrm{m}=\rho_{\mathrm{o}} \mathrm{V}_{\mathrm{o}}=65.486$ grams, $\rho_{\text {final }}=m / V_{\text {final }}=142.688 \mathrm{~kg} / \mathrm{m} 3$. The following constraint for the polytropic process condition (taken from the development outline previously in the methodology section of this paper) was programmed into MathCAD

$$
\begin{aligned}
& \frac{\left(n-2 k_{a v g}+1\right)}{(1-n)}\left[\left(2 \zeta x_{f}+1\right)^{1-n}-1\right] \\
& =\frac{2 \rho_{o}\left(u(\rho, T)-u_{o}\right)\left(k_{a v g}-1\right)}{P_{o}}
\end{aligned}
$$

Where an initial guess is used to seed the NIST SCO2 database lookup function, $u(\rho, T)$. Equation (37) was used in conjunction with the following statement of continuity for the polytropic pressure model (also taken from the development outline previously in the methodology section of this paper)

$$
P(\rho, T)=P_{o}\left(\frac{V_{o}}{V_{f}}\right)^{n}
$$

Where $P(\rho, T)$ is a NIST SCO2 database lookup function. MathCAD solves Eq. (37) and Eq. (38) iteratively until convergence is reached. Typical computational convergence for determining $n$ and $T_{\text {final }}$ is illustrated in Table 1.

Table 1. Convergence of Nominal Polytropic Index

\begin{tabular}{llllll}
\hline Iteration & $\begin{array}{l}T \\
(\mathrm{~K})\end{array}$ & $\begin{array}{l}P \\
(\mathrm{MPa})\end{array}$ & $\begin{array}{l}T_{\text {error }} \\
(\%)\end{array}$ & $\begin{array}{l}P_{\text {error }} \\
(\%)\end{array}$ & $k_{\text {avg }} / n$ \\
\hline 1 & 436 & 12.4 & 8 & 35 & $1.580 / 1.302$ \\
2 & 420 & 11.0 & 4 & 20 & $1.600 / 1.304$ \\
3 & 405 & 9.2 & 0.02 & 0.02 & $1.615 / 1.306$ \\
\hline
\end{tabular}

Affording $k_{\text {avg }}=1.615, n=1.306$. The convergence error between each iteration is listed in Table 1 as $T_{\text {error }}(\%)$ and $P_{\text {error }}(\%)$. These error criteria are monitored during the analysis in order to ensure that the $k_{\text {avg }}$ and $\mathrm{n}$ values are indeed correct for the stated assumptions. The numerical error by using this procedure is limited by the accuracy of the NIST REFPROPS SCO2 database, which is taken to be $20 \%$ for worst case conservatism. For the proceeding parameters, the energy transfer ratio from Eq. (3) is $\tau=0.503$. The cycle specific internal energy state points are $u_{\text {entry }}=521 \mathrm{~kJ} / \mathrm{kg}$ and $u_{\text {exit }}=489 \mathrm{~kJ} / \mathrm{kg}$, respectively. The mass of the $\mathrm{SCO} 2$ cycle is $\mathrm{m}=65.6$ grams. The energy per cycle is $W_{\text {cycle }}=1.277 \mathrm{~kJ}$ (1.210 BTU) given by Eq. (39)

$W_{\text {cycle }}=\frac{m\left(u_{\text {exit }}-u_{\text {entry }}\right)}{\tau+1}$
While the heat transfer per cycle is given as $Q_{\text {cycle }}=0.693$ $\mathrm{kJ}$ (0.657 BTU) per Eq. (40) given by

$Q_{\text {cycle }}=\tau W_{\text {cycle }}$

The final value of $k_{\text {avg }}=1.615$ is in comparison to the ideal gas based reference value taken at $300 \mathrm{~K}$ of $k=1.289$ [12]. Thus, if one were to employ the ideal gas value to model the elevated $\mathrm{SCO} 2$ expansion process of interest here, one would incur $25 \%$ error. Clearly, there is a difference in the $k$ value at the elevated pressures and temperatures being studied in our $\mathrm{SCO} 2$ expansion process. Monitoring the development of the primitive thermodynamic variables $T, p$, $\rho$ versus stroke during the expansion process affords Figures 5-7. Figure 5 shows the evolution of the pressure field as the piston moves the range of its stroke $0<\mathrm{x}<$ $0.0508 \mathrm{~m}(0<\mathrm{x}<2$ inches $)$. Figure 6 shows the temperature variation during the expansion process as the piston moves the range of its stroke $0<x<0.0508 \mathrm{~m}(0<x$ $<2$ inches). Figure 7 shows the evolution of the density field as the piston moves the range of its stroke $0<x<$ $0.0508 \mathrm{~m}(0<x<2$ inches $)$.

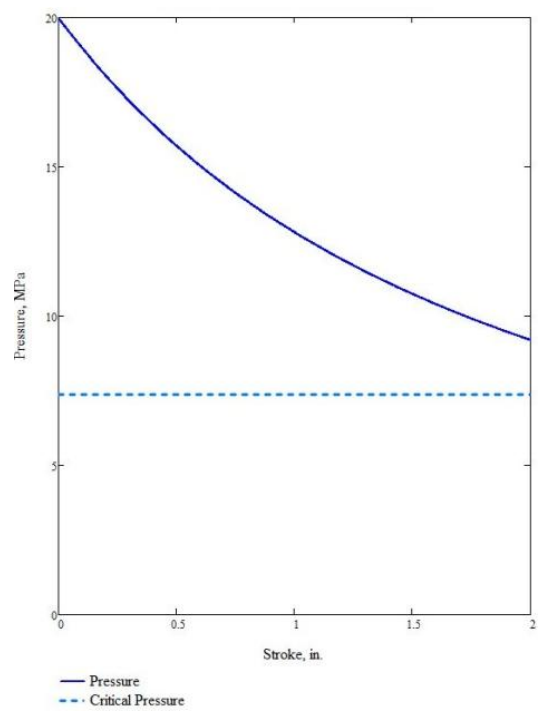

Figure 5. Pressure as a function of stroke during expansion process.

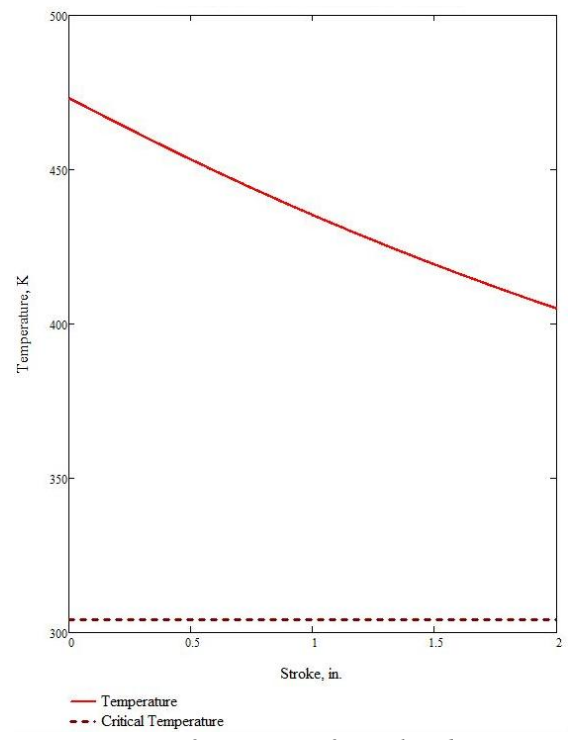

Figure 6. Pressure as a function of stroke during expansion process. 


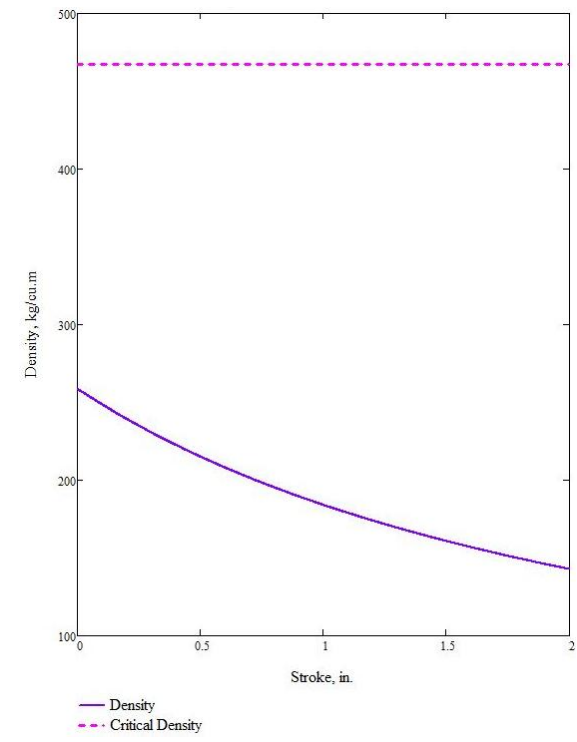

Figure 7. Density as a function of stroke during expansion process.

The dashed lines in Figures 5-7 represent the critical value of the property plotted. The significance of these dashed lines shows that the $\mathrm{SCO} 2$ does not fall below the supercritical point during the expansion process, i.e. our design of the waste heat recovery cycle assumed that the cycle remained supercritical, this is reaffirmed by inspection of Figure 7, where we see that the flow remains single phase above the supercritical point. It is important to note that from Figures 5-7 it is clear that the expansion process does not follow a simple ideal gas model, and that the polytropic expansion index is critical in determining the correct engineering physics of such complicated devices as SCO2 expanders.

The current findings of our research are in qualitative and quantitative agreement with previous studies of [1519]. When comparing our current findings of $11 \%$ efficiency it should be kept in mind that herein we have assumed a nominal pinch-point in our heat exchanger on the order of $10 \mathrm{C}$ in comparison to a pinch-point of $5{ }^{\circ} \mathrm{C}$ assumed in $[15,18]$. Our efficiency of $11 \%$ for the cycle analyzed herein agrees with the findings of [18] where high-side and low-side pressures of 200 bar, and 60 bar afford an efficiency of $13 \%$. Furthermore, our current findings agree with the high pressure limiting behavior of efficiency for $\mathrm{SCO} 2$ regenerative waste heat recovery cycles reported by $[12,13]$, where efficiencies on the order of $9 \%$ are reported for high side pressures of 150 bar. Finally, the asymptotic trends of cycle efficiency versus $\mathrm{SCO} 2$ mass flow rates reported in [19] asymptotically limit the cycle efficiency to $10 \%$ which is in qualitative agreement with the current findings of this paper. With the above comparison of our results to available data in the literature, the accuracy and validity of the proposed method has been evaluated.

\section{Conclusions}

Despite the model limitations due to the assumptions made, the polytropic process remains an accurate representation of real world piston cylinder device behavior. If one chooses to adopt the pressure model, one implicitly accepts the assumptions made about the system that entail a polytropic process. It therefore would follow that one should adopt the other implications the model has about the system, throughout its design. The method developed has been used to predict an optimal value of the polytropic expansion coefficient for a SCO2 Heat Recovery Waste Engine employing an expansion engine modeled as a polytropic process. The iterative process yields a value of $n$ $=1.306$ for the optimal polytropic expansion coefficient. The procedure also afforded a value of $k=1.616$ for the specific heat ratio of the $\mathrm{SCO} 2$ expansion process under consideration. Future work would necessarily involve testing the $\mathrm{SCO} 2$ expansion process, beginning with a value of $n=1.306$ to guide the experimental set-up. The actual value of $\mathrm{n}$ could then be determined empirically from the experimental data.

\section{Acknowledgements:}

The authors wish to thanks Butte Industries and Dr. Angela Shih, Chair of Mechanical Engineering at Cal Poly Pomona for sponsoring this research.

\author{
Nomenclature \\ $P$ pressure $(\mathrm{MPa})$ \\ $v$ specific volume $\left(\mathrm{m}^{3} / \mathrm{kg}\right)$ \\ $V$ volume $\left(\mathrm{m}^{3}\right)$ \\ $n$ polytropic index \\ $k$ heat capacity ratio \\ $\tau$ energy transfer ratio \\ $x$ troke distance $(\mathrm{m})$ \\ $A$ piston cross-sectional area $\left(\mathrm{m}^{2}\right)$ \\ $\zeta$ bore to top dead center volume ratio \\ $W$ work $(\mathrm{kJ})$ \\ $s \quad$ stroke length (m) \\ $F$ force $(\mathrm{N})$ \\ $Q$ heat $(\mathrm{kJ})$ \\ $U$ internal energy $(\mathrm{kJ})$ \\ $u$ specific internal energy $(\mathrm{kJ} / \mathrm{kg})$ \\ $m$ mass $(\mathrm{kg})$ \\ $\rho$ density $\left(\mathrm{kg} / \mathrm{m}^{3}\right)$ \\ $\alpha$ thermodynamic function (\%) \\ $\beta$ modified stroke $(\mathrm{m})$ \\ $\varphi$ modified stroke (m) \\ $\Omega$ internal energy function $(\%)$ \\ $\theta$ generic thermodynamic property \\ $X$ generic thermodynamic state \\ $Y$ generic thermodynamic state \\ $Z$ generic thermodynamic state
}

\section{Subscripts}

$\begin{array}{ll}c & \text { critical } \\ f & \text { fluid } \\ o & \text { reference state } \\ \text { avg } & \text { average } \\ \text { entry } & \text { entry state } \\ \text { exit } & \text { exit state }\end{array}$

\section{Acronyms}

BDC - Bottom Dead Center

EOS - Equation of State

NIST - National Institute of Standards and Technology

$\mathrm{SCO} 2$ Supercritical Carbon Dioxide

TDC Top Dead Center

\section{References:}

[1] Anderson, K., Devost, M., Wells, T., Forgette, D., Okerson, R., Stuart, M., Cunningham, M. Waste Heat 
Energy Regenerative Supercritical Carbon Dioxide Rankine Cycle Thermodynamic Analysis and Design. Adv. Renewable Energy, ARE-2013-11-291 Feb. 2014.

[2] Anderson, K., Clark, A., Forgette, D., Devost, M., Okerson, R., Wells, T., Cunningham, S., Stuart, M. (2013) Analysis and Design of a Lightweight High Specific Power Two-Stroke Polygon Engine. J. Eng. Gas Turbines Power 136, 041508 (Dec 12, 2013)doi:10.1115/1.4026049.

[3] Heiser, W. , Huxley, T. , \& Bucey, J. (2011). The Brayton Cycle Using Real Air and Polytropic Component Efficiencies. J. Eng. for Gas Turbines Power, 133, 111702-111710, doi: 10.1115/1.4003671.

[4] Lapuerta, M. , Armas, O., Molina, S. Study of the Compression Cycle of a Reciprocating Engine through the Polytropic Coefficient. Applied Thermal Engineering, 23, 313-323, 2003.

[5] Zhao, W. , Ye, Q., Meng, G. Measurement of Flow Rate Characteristics of Pneumatic Components Based on the Dynamic Regularity of Polytropic Exponents. Flow Measurement and Instrumentation, 22, 331-337, 2011.

[6] Chang, H. , Zhang, Y., Chen, L. An Applied Thermodynamic Method for Correction of TDC in the Indicator Diagram and its Experimental Confirmation. Applied Thermal Engineering, 25, 759$768,2005$.

[7] Stone, R. The Internal Combustion Engine, SAE Press. 2013.

[8] Heywood, J. Internal Combustion Engine Fundamentals. New York, NY, McGraw-Hill, 1998.

[9] Faires, V. Thermodynamics, (6 $6^{\text {th }}$ ed.). New Jersey, Macmillan, 1978.

[10] Taylor, C. The Internal Combustion Engine in Theory and Practice, Volume 1: Thermodynamics, Fluid Flow, Performance ( $2^{\text {nd }}$ ed.). Cambridge, MIT Press, 1985.

[11] Christians, J. Approach for Teaching Polytropic Processes Based on the Energy Transfer Ratio. Int. J. Mechanical Engineering Education., 40, 53-65, 2012.
[12] Cengel, Y. \& Boles, M. Thermodynamics an Engineering Approach. New-York, McGraw-Hill, 2011.

[13] E.W. Lemmon, M.L. Huber, M.O. McLinden, "NIST Standard Reference Database 23: Reference Fluid Thermodynamic and Transport Properties-REFPROP”, National Institute of Standards and Technology, Standard Reference Data Program, Version 9.0, 2010.

[14] http://www.ptc.com/product/mathcad/

[15] Chen, H., Goswami, D.Y., Stefanakos, E. A Review of Thermodynamic Cycles and Working Fluids for the Conversion of Low-Grade Heat, Renewable and Sustainable Energy Reviews, 14, 3059-3067, 2010.

[16] Cayer, E., Galanis, N., Desilets, M., Nesreddine, H., Roy, P. Analysis of a Carbon Dioxide Transcritical Power Cycle Using a Low Temperature Source, Applied Energy, 86, 1055-1063, 2009.

[17] H. Tou, "Parametric Analysis of a Reheat Carbon Dioxide Transcritical Power Cycle Using a Low Temperature Heat Source", 2nd International Conference on Environmental Engineering and Applications, IPCBEE, Vol. 17, 2011, IACSIT Press, Singapore, 2011.

[18] Y. Chen, "Novel Cycles Using Carbon Dioxide as Working Fluid - New Ways to Utilize Energy from Low-Grade Heat Sources, Thesis, School of Industrial Engineering and Management", Dept. of Energy Technology, Division of Applied Thermodynamics and Refrigeration, Stockholm, Sweden, May 2006.

[19] Chen, Y., Lundqvist, P., Johansson, A., Platell, P. A Comparative Study of the Carbon Dioxide Transcritical Power Cycle with an Organic Rankine Cycle with R123 as Working Fluid in Waste Heat Recovery, Applied Thermal Engineering, 26, 2142-2147, 2006. 